\title{
Twisted Logic | Thinking Outside and Inside the Box
}

\author{
BLAIR SATTERFIELD \\ University of British Columbia \\ DEREK MAVIS \\ University of British Columbia
}

\author{
ALEXANDER PREISS \\ University of British Columbia \\ GRAHAM ENTWISTLE \\ University of British Columbia
}

\section{INTRODUCTION | SMALL MATERIAL MOVES CAN BEND LARGE SYSTEMS}

Light frame construction with wood is flexible, adaptable, cheap, renewable, and requires very little skill to assemble. It is a dominant system for good reason and has changed little in over a century. We believe introducing our project titled "Zippered Wood" to the stick framing approach could reframe this construction method, making it more responsive and resource responsible. We are developing a pair of experimental wall prototypes that challenge conventional wall construction through the free modification of its most basic component, the $2 \times 4$. Our investigation works at the scales of wood grain, wood member, and software interface to free the architect to conceptualize and deliver more sophisticated wood framed buildings. We aim to empower the architect by amplifying her control over material forming strategies, introducing adaptability into the forming process, and finally reducing the cost of shaping materials in time, treasure, and setup. We have installed a wall prototype to test the strategy, and at the time of this publication, the team is working toward a pavilion-scale prototype. These projects are discussed at the end of this paper. Before we discuss pavilions we will outline and situate the material innovations, the software we are developing to allow architects to apply those innovations, and context for our work.

\section{IMMATERIAL FORCES IMPACTING THE MATERIAL WORLD | WORKING IN THE WAKE OF GLOBAL CAPITAL}

At times it seems as though architects have remarkably little agency in making the urban fabric we help design. The fiscal power and market politics of global capital conspire to diminish our influence. The voices of academic researchers feel even fainter when railing against the forces of asset urbanism. In a city like Vancouver Canada, the ideas of "materiality and methodology" in architecture, while present and important, are far from the heart of public conversations about buildings, building, and city making. Each is present of course but discourse and interest veer toward marketability, housing availability, and workflow efficiency. A trend some architects resist, crafting elegant exceptions to the fiscal rules of the new urban realm. However, many more fall in line, dressing developer pro forma in marginally expressive facades and marketable finishes.
From this vantage point, the challenge for twenty-first century architects is finding places to apply effective pressure to an immaterial body of economic forces. Where can architects make any meaningful impact in this space? We decided to look for material opportunities in the wake of global capital.

Metro Vancouver is a dense, geographically bounded urban region that exists largely on the Burrard Peninsula and the southern edge of the Coastal Mountain Range. A more suburban fabric sits on the Fraser River delta and now sprawls up the Fraser Valley towards the northern tip of the Cascade Mountains. The Lower Mainland boasts Canada's most temperate climate and abundant natural beauty. It has a sheltered bay and harbour, beaches and skiing, fresh water, clean air, and a diverse population. These factors have helped transform Vancouver from a wilderness hub for resource extraction and Canada's western seaport, into one of the hottest (and most expensive) real estate markets on the planet. The city has become a fiercely sought global asset, traded, hoarded, and flipped by moneyed speculators. This problem is only getting worse. A recent study titled "Metro Vancouver 2040 "1 predicts that one million people will be added to the region in the next 25 years. This represents a $41 \%$ increase in population. Most of that growth is going to be accommodated in existing neighborhoods and will be achieved through the demolition of older single-family houses and other light frame buildings.

One outcome of these changes is and will continue to be an abundance of construction waste. In 2015 alone the construction industry in Metro Vancouver produced approximately 218,000 tons of waste wood including $29 \%$ untreated dimensional lumber. ${ }^{2}$ Throughout North America, it is estimated that roughly $20 \%$ of all materials that enter landfills came from construction and demolition waste. Put more bluntly, in Vancouver and across North America, the effects of a series of abstractions (soaring land values, lax oversight on real estate speculation, and construction undertaken to shelter and launder money) contribute to the destruction and disposal of nearly 1000 single-family homes annually ${ }^{3}$. These older buildings contain wood that is serviceable and in many cases superior in quality to new timber members. Salvaged $2 \times 4 s(2 \times 6 s, 2 \times 8 s . .$.$) are often from$ 


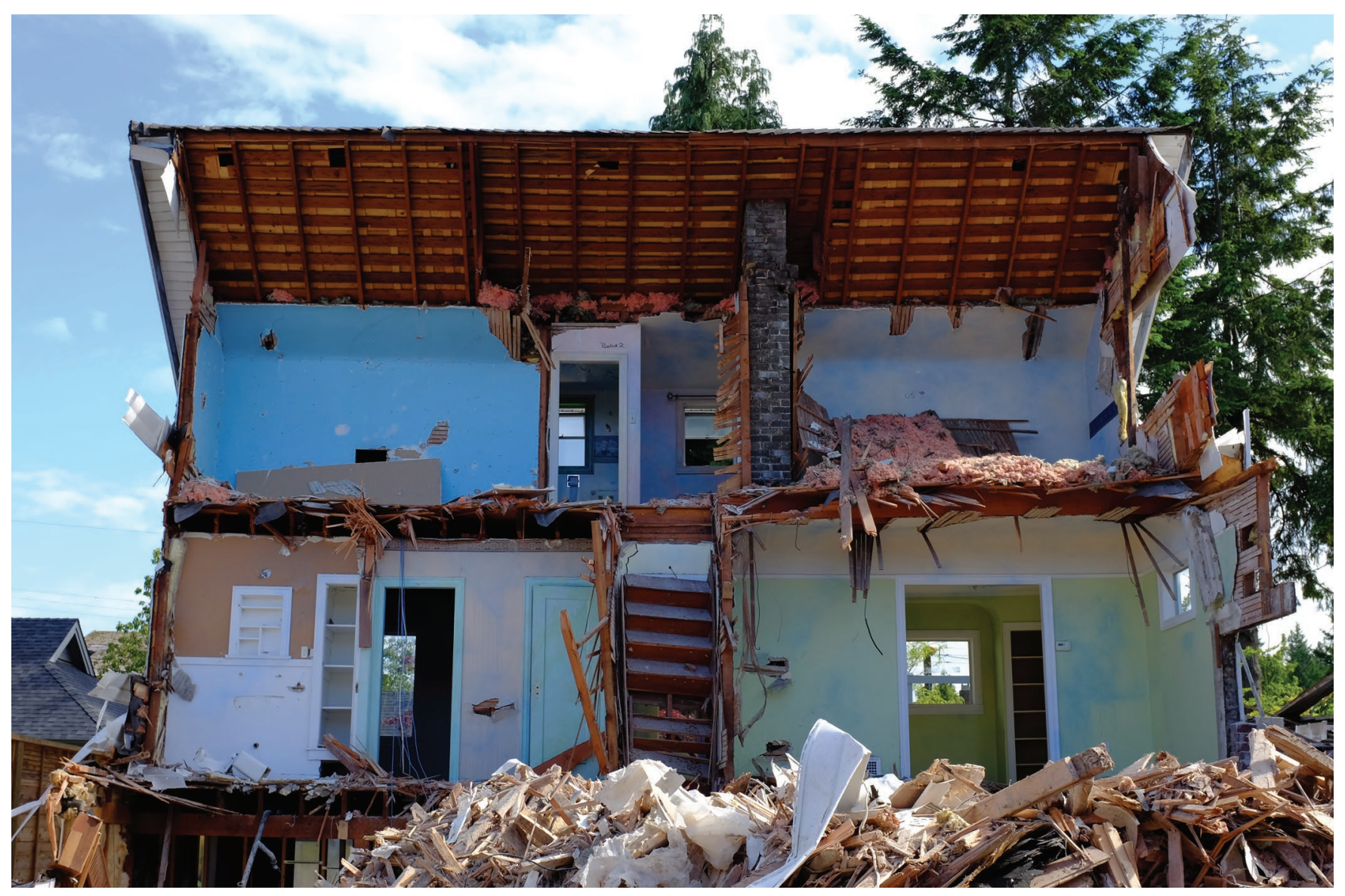

Figure 1. Approximately 1000 single family homes are torn down annually in Vancouver proper (statistics gathered from 2009 forward).

The majority of material from this demolition ends up in a landfill. Image provided by authors. Statistics based on demolition permits issued by the City of Vancouver over the last six years.

older growth trees, are dry and therefore dimensionally stable, and may be longer and more robust than today's standard eight-foot stock. Much of this waste material is still viable for light wood frame construction.

A portion of our research focuses on the upcycling of reclaimed materials. This includes dimensional lumber with particular attention paid to the ubiquitous $2 \times 4$. Our Zippered Wood process (presented in this paper) is a novel take on wood joinery and deformation in which digitally generated formally specific "joint patterns" are cut into boards that are joined to produce predictably precise bends. Our approach takes its queues from projects that generate form using material behavior and smart geometry. We are advancing those techniques from analog production to digital, and from precision fitting to precision displacement. To achieve this, we have developed software strategies that allow us to simulate a desired material bend in virtual design space and calculate the mathematically specific "tooth" pattern necessary to make that exact bend. Once generated, the pattern is output to toolpaths that are fed into a CNC mill and used to modify waste timber members. When zipped together, these members generate the predicted twists and curves of the simulation.

\section{CREATING NON-ORTHOGONAL ARCHITECTURAL ASSEMBLIES USING THE MOST COMMON LINEAR BUILDING COMPONENT (THE 2X4)}

The "Zippered Wood" project began as part of a larger challenge. Our research group is currently working to digitally fabricate and erect freestanding pavilions made entirely out of waste material (figure 5). These projects convert plastic, fabric, and wood waste into structure, connectors, and skins using CNC mills and custom fabrication tools and processes. This paper focuses specifically on one aspect of this larger research effort; the transformation of wood waste in the form of salvaged lumber. Using seed funding for a first summer of inquiry, we explored how to turn short lengths of waste $2 \times 4$ (breakage and scrap) into long, curved posts and beams able to integrate into wood framed buildings. Our first attempts used a traditional approach; gluing thin veneers cut from lumber over complex (and wasteful) formwork to create bent shapes. We quickly learned two lessons: first, converting $2 \times 4$ s into veneers had a roughly $50 \%$ waste factor. Secondly, and predictably, formwork quickly became a limiting factor. A project with any degree of complexity requires many distinct pieces. This in turn requires the production of numerous unique cauls with which to glue veneers. It quickly became 


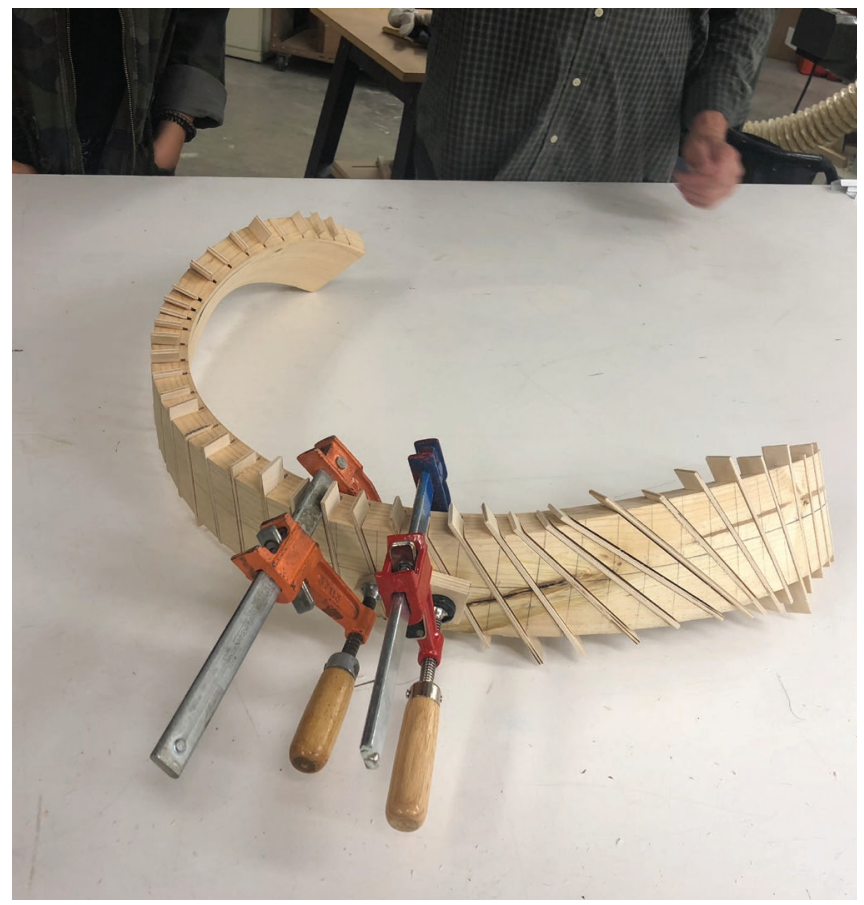

Figure 2. Kerfed Prototype demonstrating surface rulings. image provided by authors.

apparent that our process was inefficient with both material and time. We were generating too much waste in our effort to transform and eliminate waste.

It was while modifying formwork (using kerfed boards for cauls) that we hit upon a very interesting idea. What if we were able to strategically modify $2 \times 4$ s with specific geometries (joints in the form of teeth) and mate them to generate a bent form? The modified boards could "zipper" together, forcing predictably bent and twisted composite $2 \times 4$ s. Assembly of these members could be accomplished with no formwork, just pure geometry producing its own expression. This idea led our team to examine non-orthogonality (twisting and bending) as a strategy. Bending and twisting are emergent material behaviors of wood that are typically avoided in traditional light-frame construction, yet wood's capacity to bend without the collapse of its internal structure makes it an ideal material for non-orthogonal assemblies. We believe there is potential in the bend, and became interested in how we might transform wood "sticks" salvaged from linear structural systems into curvilinear members. Upcycling allows a single piece of wood to exist with three radically different identities. The original board capitalizes on its end bearing capacity. The transformed version exploits its internal bending capacity. Both are generated from a piece of a larger structure, the tree.

Our "eureka" moment shifted the focus of our work from lamination to calculation. We began developing analog versions of our zippered strategy. Bending was initially done using wedges to generate curls and twists in stock material. Our ability to manually generate geometry was encouraging but too unpredictable and imprecise. It also left us with formed members that were structurally compromised because the mating surfaces were too inexact. While working on the process it occurred to us that we needed a new method for formally modifying the $2 \times 4$ members. We needed more control and better surfaces for gluing modified pieces of $2 \times 4$ back together. In response, the team began developing digital scripts in Grasshopper. The goal was to take a designed shape (a virtually twisted $2 \times 4$ member specified by an architect for example) and digitally generate a unique "tooth" pattern that would result in a bend when two unique halves are "zippered" together. While working on the project we found material and digital precedents that informed the crafting of our project.

\section{FORMING WITHOUT FORMWORK - MATERIAL PRECEDENTS}

Boat builders are common in the Pacific Northwest and Lower Mainland. Production varies in scale from shipyards producing commercial steel vessels to individuals building small paddle craft. John Lockwood is an example of the latter. $\mathrm{He}$ is the founder of Pigmy Sea Kayaks in Port Townsend, Washington. Lockwood is interesting to us for two reasons. First, his technique for boat building used no formwork. Second, he developed his own software to produce templates that informed his material craft. Lockwood claims to be the first person to use computer software to design and build "stitch-and-glue" wooden kayaks. ${ }^{4}$ His boats are built from wood sheets that are cut with specifically designed edge profiles. Initially these profiles were cut manually but they have since been generated using a CNC mill. Once cut, the aptly named Lockwood sews the wood veneers together. This forces the wood into shape. Once all the pieces are sewn and the kayak's shape resolved the assembly is fixed with glue, the stitching is removed, and the boat is finished.

Lockwood's edge-formed compound geometries are useful examples of leveraging material behavior and geometry to make form. It also shows a natural relationship between analog and digital craft. While enlightening, the Pygmy example is not a template for transforming $2 \times 4 \mathrm{~s}$. For clues on how to transform a solid block, the team looked to a method called 'ZipShape' developed in Germany by Christoph Schindler. ${ }^{5}$ Schindler's digital kerfing strategy uses 3D modeling software and 6-axis robots to generate meshing panels that, when locked together, form undulating surfaces. Schindler applies his technique to furniture, cutting his kerfs out of a soft core faced with wood laminate. His techniques generate planar curves along a single axis. Kennedy and Violich Architecture advance this technique with "Smart Rockers," a clever public furniture project that uses small Kuka robots to mill MDF cores. When assembled, the sheets of laminated MDF achieve sweeping axial bends. All three precedents offer 


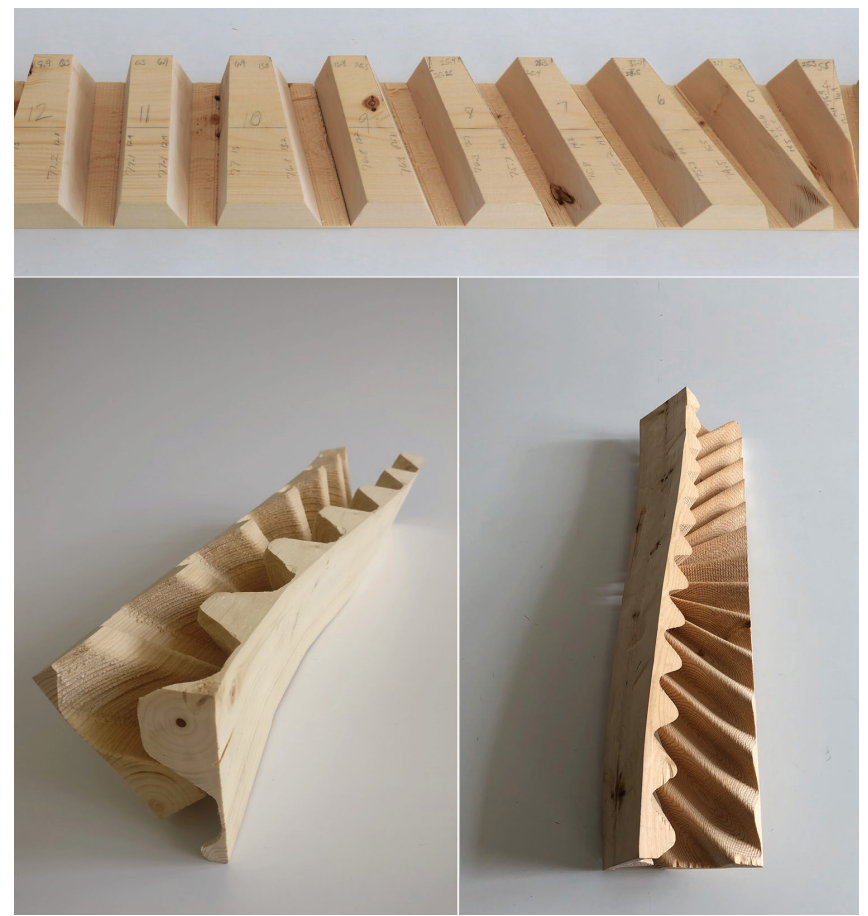

Figure 3. Prototypes of zippered wood with prismoidal teeth made with cross-cut saw (top), CNC milled faceted teeth (bottom left), and sinusoidal teeth (bottom right). image provided by authors.

valuable insights into the generation of form using material behavior and smart geometry, but work was still necessary to achieve our objective.

\section{PHYSICAL AND DIGITAL SIMULATION - PARALLEL PROCESSES PROGRAMMING SOFTWARE AND MATERIAL FORM}

A "kerf" is a groove made by removing material with a cutting tool (saw, torch, bit, etc.). "Kerfing" can be defined as the act of making repeated parallel shallow cuts through a solid block to allow that modified block to bend. A $2 \times 4$ can be made remarkably flexible through kerfing, but the bending is unpredictable and the resulting board is structurally diminished. These complications prompted our decision to change direction and focus on controlling the cut. Our kerf cuts went from parallel to eccentric. They then morphed into modified finger joints and eventually evolved into continuous topographies able to predictably deform material (figure 2).

Our research strategy informed this evolution. We divided our team, assigning one group physical prototypes and the other virtual models. Both teams consulted frequently. The material team started with prototypes that were hand measured and milled from $2 \times 4$ s using a compound mitre saw to produce examples with polyhedral teeth. These prototypes were trained together to form bends and twists. The results were encouraging. We had successfully modeled our approach physically. The other team's task, bending $2 \times 4$ s virtually using
Rhino and Grasshopper, was more straightforward. The gap in the process was in translation from physical to virtual. To bridge it we developed Grasshopper scripts to analyze our simulated shapes and to generate the toolpaths required by a CNC mill to produce viable tooth patterns.

Outputting the digital model revealed new challenges. First, the depth of the cuts affected the wood's ability to bend. Too much wood left at the thinnest point of a given "valley" cracked due to material stiffness. Too little wood failed due to weakness. To find an optimal thickness, we iteratively cut a series of physical prototypes using a consistent tooth pattern. We started with a $6 \mathrm{~mm}$ maximum depth and decreased through subsequent millruns. Our most successful test prototypes left a minimum of $3 \mathrm{~mm}$ of wood along the flat face of the milled board. When tested, this piece was both flexible enough to be formed yet proved very stiff once glued. The assembled member was able to resist twisting and bending forces. The second challenge was the gluing surface itself. Our faceted cuts exposed too much end grain, which is poor for gluing, and the interior corners of the faceted teeth were prone to cracking under loading (figure 3 ).

One solution was to smooth the tooth profile. We developed an undulating sinusoidal pattern that generated a continuous gluing surface (figure 3 , sine wave). The glued bond was much better than anything we achieved with faceted cuts. The sinusoidal strategy showed us that the geometry imbedded in the angle of the teeth was the defining factor in predictably bending wood (not the shape of the tooth). We have been progressing a planar strategy in parallel to the sinusoidal work. The planar approach dramatically reduces milling time. Finally, we applied more traditional dovetail joints to end join already formed boards. The combination of these techniques meant we could take breakage and off-cuts and theoretically generate articulated boards at any length, be they straight, twisted, curved, or bent.

\section{BREAKING THE BLACK BOX | BENDING SIMULATION IN GRASSHOPPER AND REVERSE ENGINEERING DEVELOPABLE SURFACES}

Successfully translating software-generated sine waves into physical assemblies gave us the confidence to speculate on application and to refine our digital approach. Our bending simulation is built primarily in the Rhinoceros 6.0 modeling environment and scripted using Grasshopper and Kangaroo. Several precedents have been instrumental in progressing our work in this space. Daniel Piker's Kangaroo plug-in for Grasshopper is the physics engine that allowed us to work with forces in real time. ${ }^{6}$ Merten Nettelbladt demonstrated that a Kangaroo simulation of a spring network could result in a developable surface (defined in differential geometry as "a three dimensional surface that can be mapped onto a plane without any distortion." ${ }^{\prime 7}$ ) Following Nettelbladt's example allowed us to resolve $2 \times 4$ s of any given length between any 

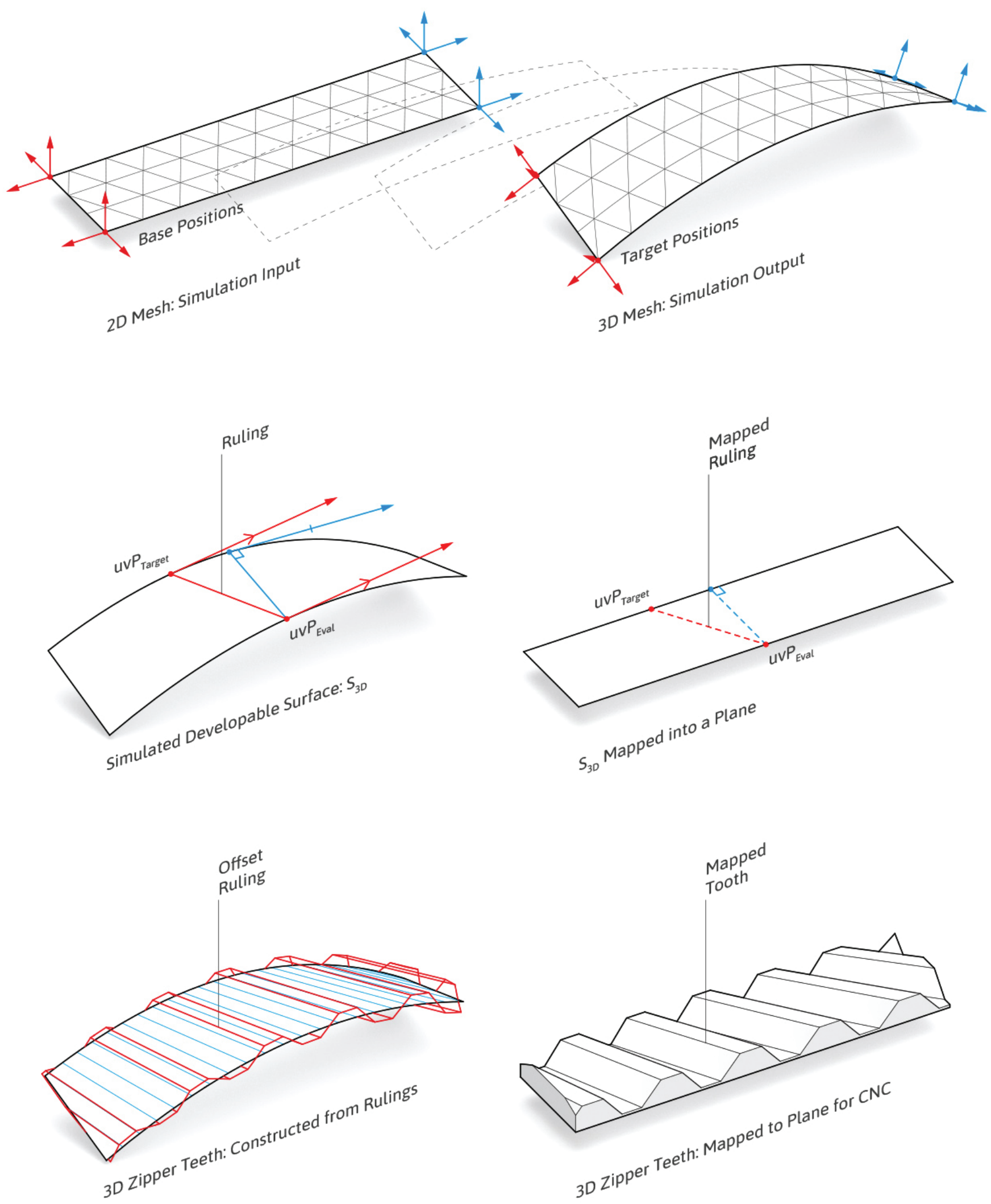

Figure 4. Graphic description of tooth pattern generation. 
paired end conditions, (figure 4) and to unroll a figural 3D surface into a $2 \mathrm{D}$ rectangle with the same proportions as a $2 \times 4$. This unrolling is akin to manually forcing a loop made from a paper strip to lie flat. Our objective was to generate a developable surface for our project. Without this key ability, any bending we aimed to re-create in wood would not be accommodated by linear pieces.

The inputs of this simulation are a $2 \mathrm{D}$ spring network, the location and orientation of its flat ends, and the goal location and orientation of each end once transformed. Kangaroo was instructed to maintain the starting length of each spring and to strive for an angle of 180 degrees between each polygon denoted by 3 springs. When activated, the script moved the ends of the spring network from its 2D positions to its 3D positions. Kangaroo's iterative solver updated the locus of this spring network throughout this transformation. The result was an approximation of a 3D surface that maintained the same surface area and proportions whether flat, bent, or twisted. From this we were able to know the length of any $2 \times 4$ required to make a given transitional geometry. In essence, the software generated a flexible virtual $2 \times 4$. This is useful for output and input, allowing designers to update and iterate the design of these virtual components in real time. We were also assured that in principle the form produced virtually was possible to construct out of rectangular stock.

While the simulation of a developable surface was an important step in our software development, it was not useful until we could successfully analyze its 3D curvature to inform the geometry of a tooth pattern. This is a relatively straightforward problem to solve when using simple developable surfaces, like those found on a cylinder or cone (found in our precedents: "ZipShape" and "Smart Rocker.") It becomes more complex when the surfaces are not mathematically derived from a primitive solid, as they require the designer to pre-determine the desired shape before solving.

Knowing the location and orientation of rulings is crucial in bending. The team gained its understanding of ruling angles and their agency through physical models. It was simple to iteratively and spontaneously vary the angle of a relief cut in a section of $2 \times 4$ (figure 2). The block form would twist or bend according to the angle of each cut. This bottom-up approach was useful but limiting, specifically because we did not possess a reliable way to anticipate the final position of the entire assembly, because each bend has a cumulative dislocating effect on the location of the next section of board. We could make and read cuts, but we needed a way to infer the position of each ruling based on the simulated surface curvature alone. We needed a top down approach.

We identified three critical rules when generating the solution to this problem. First, for a developable surface with two parallel edges, the required rulings will traverse at a right angle across the face from one edge to the opposite edge unless the surface is bending or twisting about its long axis. Secondly, if we were to loft between each ruling on the 3D surface, the resulting lofts would be expected to be planar. ${ }^{8}$ Finally, because our simulated surface is a rectangle with two parallel edges, and that upon unrolling that surface we expect to see a uniform rectangle footprint, we can infer that the tangencies at the two points where a single ruling intersects each 3D edge curve will be parallel (figure 4). These rules informed and limited our scripted analysis.

For any given point evaluated on one 3D edge of our virtual $2 \times 4$, the script compares its tangency to thousands of sample points on the opposite target edge. When the nearest match is found, the system refines the search. A single line connects the two resulting points found to have parallel tangents. The position of this line and its angle relative to the surface is the appropriate ruling for that location. That line is the rotational axis for the expected curvature. This process was repeated along the face of the curved 3D surface. Lastly, the script generates a tooth-pattern from the cumulative set of rulings. Adding or subtracting newly derived rulings adjusted the pattern's frequency. The result was gear-like teeth. We have been testing and tweaking the variables between input, output, and fabrication since this breakthrough was made.

\section{FORECASTING FUTURES - FUTURE BUILDING AND RESEARCH}

One current focus of exploration is the research and development of tools. We are in the early stages of designing a portable machine that will simultaneously roll and glue sections of zippered wood together. We aim to push the joined members through an RF (radio frequency) threshold to rapidly cure the glue. If successful, the entire zipping process could happen within minutes. We believe this development would support high volume production of bent dimensional lumber for on-site construction applications. We are also researching new milling tools and techniques to achieve smoother and faster cuts. On the software side, we plan to develop a plugin that will simplify the use of our software innovations.

The team is currently working on two projects that will test the Zippered Wood method at an architectural scale. Stick Formed Wall is a variation on a traditional stud wall. By curving the $2 \times 4 \mathrm{~s}$ that comprise the bottom plate of a traditionally framed wall we can transform a portion of a planer wall into a volumetric hyperbolic paraboloid. Our design "delaminates" to create a modest covered space for the display of boards explaining the Zippered Wood project. The goal of this proposal is to demonstrate that while Zippered Wood can be used in all members of a structural system (yielding highly complex forms), the system's lasting value might be found in its compatibility with existing light wood-framing techniques (figure 5). 

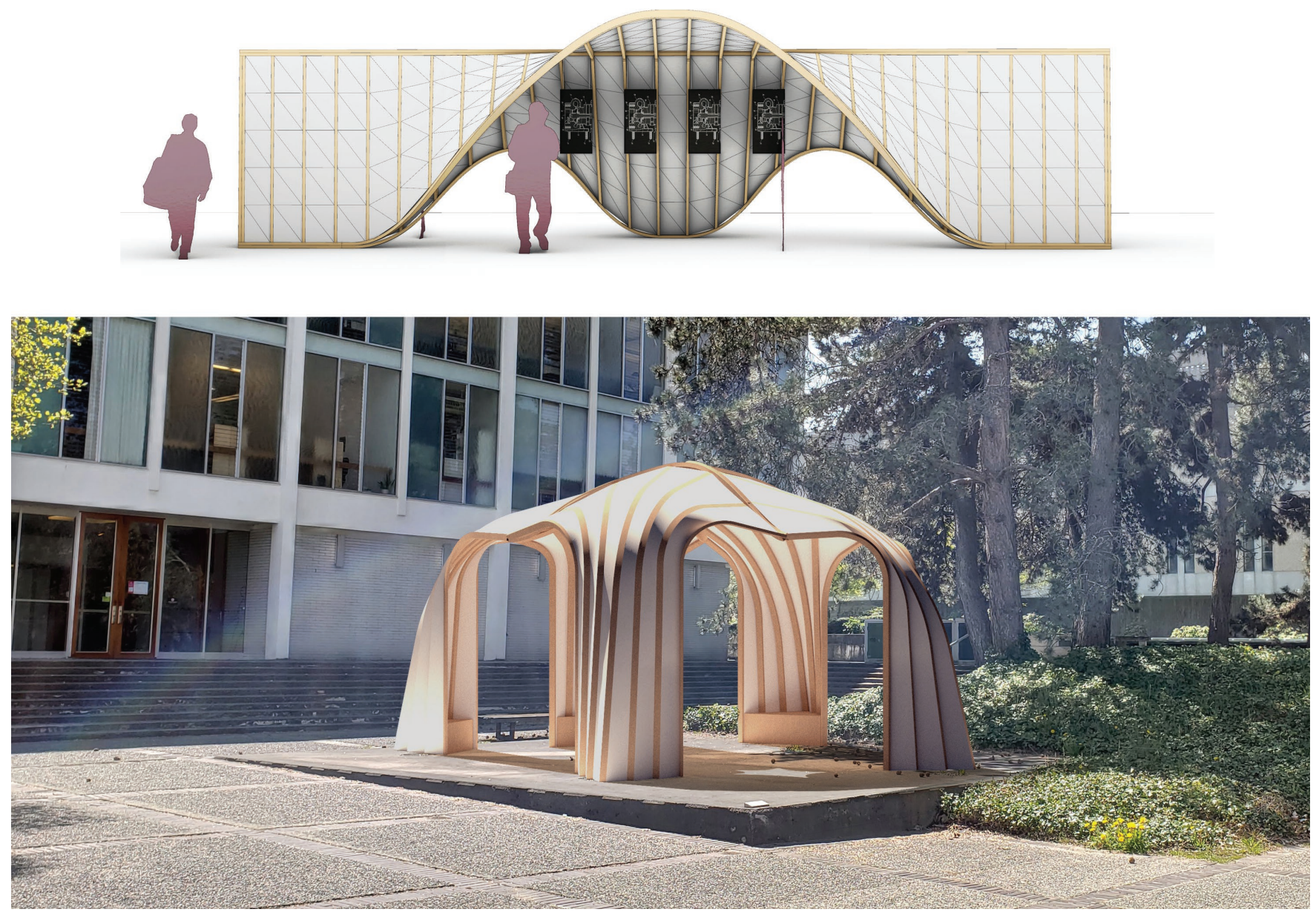

Figure 5. Pavilion prototypes: Stud Framed Wall (top) and Paper or Plastic (POP) Pavilion (bottom). Image provided by authors.

Our second prototype is more ambitious. We will explore Zippered Wood's formal capacity through the development of an outdoor pavilion designed to house student performances and impromptu teaching. Its structure and skin will be fabricated using only repurposed second source material (wood and plastic in the current design). The pavilion will sit on five existing eccentrically placed footings. Our design features bundled columns built from salvaged $2 \times 4 \mathrm{~s}$. Each bundle rests on a footing, bending and fanning inward to create cover. The five unique fans translate between irregular footing placements and a shared center. We have been looking to Pacific Coast First Nations and their rich tradition of basket weaving for precedents on how to mesh formally free linear members into a cohesive material assembly. Through this pavilion we hope to demonstrate that customization should carry no extra fabrication time or material cost. We see these projects as incremental steps in our research, as well as provocations. Our intent is to demonstrate the potential of Zippered Wood and to find optimal locations for its application.

\section{CONCLUSIONS}

It is impossible to know how architecture will weather the coming decades. The world is facing massive change, including increasingly turbulent market forces, diminishing resource availability, a transition from computerization to computational work, and inevitable automation. Perhaps automation will save us, freeing the architect to refocus on inherited roles as auteurs of form and meaning. Maybe we are already lost, the proverbial "frogs in the pot," unaware we are doomed as we yield to algorithms and robots. Most likely architecture will evolve, our techniques merging with technology, blurring the boundaries between design and building, policy and culture, material and programming. Whatever the scenario, it is clear that we can no longer be content to simply refine our methods. We must enter the black box and program it, disrupt it, and redirecting its output. The Zippered Wood process is offered as an example of how. This project is our novel take on wood joinery and material deformation, deployed to convert standard $2 \times 4$ s into formally sophisticated building components. Our approach generates form using material behavior and geometry, advancing 
techniques from analog production to digital (and back), and from precision fitting to precision displacement. We also recognize that this process results in the loss of material in modified pieces. Despite this loss, Zippered Wood positions us to utilize reclaimed material and off-cuts, thus extending the life of otherwise compromised wood members destined for the landfill. Zippered Wood could generate a new vocabulary of built form using readily accessible stock material (the $2 \times 4$ ) and waste material (salvaged lumber). This process emerged out of the constraints of existing dimensional lumber, specifically the $2 \times 4$ 's parallel edges, standard dimensions, and orthogonality. These same attributes are what make the Zippered Wood process possible. Parallel longitudinal edges make the surface rulings much easier to locate. Near continuous face grain allows for bending to occur. Our system re-imagines reclaimed lumber as an opportunity for sustainable construction that is not bound to the orthogonal status quo. We hope that by challenging the limits of recycling we might create a new urgency for reuse in architecture. Zippered Wood is a designed contagion that could be productively disruptive precisely because it simultaneously takes responsibility for the black box logics of material production and programming as it seamlessly adapts to standard construction practices. The project could be part of a larger strategy that radically changes the building industry's relationship with wood products and sustainable wood use. Our goal is to influence wooden architecture just as the straight $2 \times 4$ did over a century ago.

\section{ENDNOTES}

1 "Metro Vancouver 2040 - Shaping Our Future," Metro Vancouver, July 29, 2011. http://www.metrovancouver.org/services/regional-planning/ PlanningPublications/RGSAdoptedbyGVRDBoard.pdf.

2 Terry Fulton, "2015 Demolition, Land-Clearing, and Construction Waste Composition Monitoring Program," Metro Vancouver, January 11, 2016. http:// www.metrovancouver.org/ services/solid-waste/SolidWastePublications/2015D LCWasteCompositionMonitoring.pdf .

3 Statistic based on demolition permits issued by the City of Vancouver over the last six years.

4 John Lockwood, “About Pygmy Boats," Pygmy, 2018. https://www.pygmyboats.com/about-pygmy-boats.html

5 Christoph Shindler, "ZipShape Mouldless Bending II: A Shift from Geometry to Experience," Respecting Fragile Places: 29th aCAADe Conference Proceedings, eds., Tadeja Strojan Zupancic, et al. (Ljubljana, Slovenia: University of Ljubljana Faculty of Architecture, 2011).

6 Daniel Piker, "Kangaroo: Form Finding with Computational Physics," AD: Special Issue: Computation Works: The Building of Algorithmic Thought 83, no. 2 (March 2013): 136-7.

7 Yang Liu, et al., "Geometric Modeling with Conical Meshes and Developable Surfaces," ACM Transactions on Graphics 25, no. 3 (July 2006): 681.

8 Liu, et al., 685 . 